\title{
Effect of spatial resolution on the estimates of the coherence length of excitons in quantum wells
}

\author{
M. M. Fogler, ${ }^{1}$ Sen Yang, ${ }^{1}$ A. T. Hammack, ${ }^{1}$ L. V. Butov, ${ }^{1}$ and A. C. Gossard ${ }^{2}$ \\ ${ }^{1}$ Department of Physics, University of California San Diego, La Jolla, California 92093 \\ ${ }^{2}$ Materials Department, University of California at Santa Barbara, Santa Barbara, California 93106
}

(Dated: August 19, 2021)

\begin{abstract}
We evaluate the effect of diffraction-limited resolution of the optical system on the estimates of the coherence length of two-dimensional excitons deduced from the interferometric study of the exciton emission. The results are applied for refining our earlier estimates of the coherence length of a cold gas of indirect excitons in coupled quantum wells [S. Yang et al., Phys. Rev. Lett. 97, 187402(2006)]. We show that the apparent coherence length is well approximated by the quadratic sum of the actual exciton coherence length and the diffraction correction given by the conventional Abbe limit divided by $\pi$. In practice, accounting for diffraction is necessary only when the coherence length is smaller than about one wavelength. The earlier conclusions regarding the strong enhancement of the exciton coherence length at low temperatures remain intact.
\end{abstract}

PACS numbers: 78.67.-n,73.21.-b,71.35.-y

\section{INTRODUCTION}

Spatial coherence of a bosonic system is encoded in its one-body density matrix

$$
\rho(\mathbf{r})=\left\langle\Psi^{\dagger}\left(\mathbf{r}^{\prime}\right) \Psi\left(\mathbf{r}^{\prime}+\mathbf{r}\right)\right\rangle,
$$

where $\Psi^{\dagger}(\Psi)$ is the particle creation (annihilation) operator and the averaging is over both the quantum state and the position $\mathbf{r}^{\prime}$. In an isotropic system $\rho(\mathbf{r})$ depends on the absolute distance $r=|\mathbf{r}|$ only. The density matrix decreases with $r$ as a result of scrambling the phases of the particles' wavefunctions by scattering and thermal fluctuations. When this decrease is faster than $1 / r^{2}$, we can define a characteristic decay length of $\rho(r)$ - the coherence length - by the relation

$$
\xi_{x}=\left(\int_{0}^{\infty} \rho(r) r d r\right) /\left(\int_{0}^{\infty} \rho(r) d r\right) .
$$

Coherence length $\xi_{x}$ provides a quantitative information about fundamental properties of the system of interest. For example, a rapid growth of $\xi_{x}$ is anticipated as the bosons are cooled down below the temperature of their quantum degeneracy 1,2 In addition, $\xi_{x}$ sheds light on interactions and disorder in the system.

A gas of indirect excitons in GaAs coupled quantum wells is an example of a solid-state system where this rich physics can be studied. A number of basic physical parameters of such gases (concentration, exciton lifetime, etc.) can be controlled to bring them to a quasiequilibrium at very low temperatures ${ }^{3}$ The coherence of excitons is imprinted on the coherence of the light they emit $4,5,6,7,8$ This allows one to measure $\xi_{x}$ by optical methods. In this paper we discuss a particular real-space technique, which has proved to work in our experimental conditions $\stackrel{\underline{9}}{\underline{\underline{y}}}$ It has enabled us to determine the exciton coherence length $\xi_{x}$ and confirm its rapid increase as temperature $T$ drops below a few degrees $\mathrm{K}$.
Traditionally, real-space measurements of the optical coherence are done by two-slit (or two-point) interferometry. However, this method becomes inaccurate when $\xi_{x}$ is smaller than the spatial width of the regions from which the light is collected. This is the case in our experiment where $\xi_{x}$ does not exceed a few microns. However, our technique circumvents this limitation by using a single pinhole. It works well in the regime $\xi_{x}<D / M_{1}$, where $D$ is the pinhole diameter and $M_{1}$ is the image magnification factor. In other words, the smallest measurable $\xi_{x}$ is determined not by $D / M_{1}$ but by the finite spatial resolution of the optical system. In this paper we show how this resolution can be taken into account.

The paper is organized as follows. In Sec. [II we summarize the main elements of the experimental technique and present our principal results. In Sec. III we review the theoretical model used in Ref. 9. In Sec. IV we refine it to incorporate the finite-resolution effects. Discussion and conclusions are given in Sec. $\mathrm{V}$

\section{RESULTS}

\section{A. Measured exciton coherence length}

In order to introduce the key parameters of the problem, we need to briefly review the basic steps of the experimental implementation of our method for measuring the coherence length. ${ }^{9}$ The exciton PL is collected from an area of size $D / M_{1}=2-10 \mu \mathrm{m}$ in the middle of one of the exciton beads $\frac{10}{10}$ ranging $\sim 30 \mu \mathrm{m}$ across. This is done by placing a pinhole of diameter $D=10-50 \mu \mathrm{m}$ at the intermediate image plane of magnification $M_{1}=5$, see Fig. 1a. The light is then passed through a MachZehnder (MZ) interferometer with a tunable delay length $\delta l$. The output of the interferometer is further magnified by the factor $M_{2} \approx 1.6$ (so that the total magnification factor is $M=M_{1} M_{2}$ ) and then dispersed with a grat- 


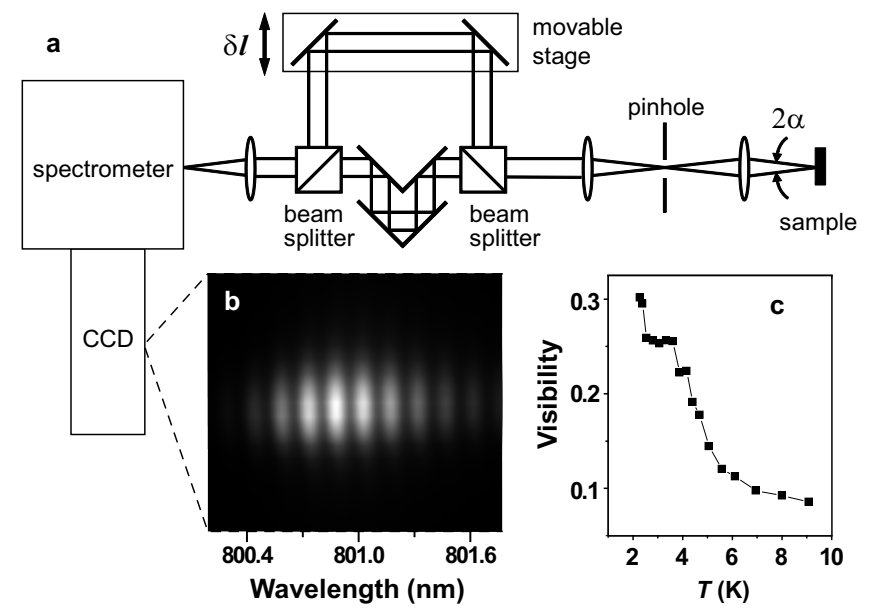

FIG. 1: (a) Experimental setup. The collection angle of the lens $2 \alpha=32^{\circ}$. (b) The interference pattern on the CCD for $D=25 \mu \mathrm{m}, \delta l=4.2 \mathrm{~mm}$, and $T=1.6 \mathrm{~K}$. (c) Visibility function $V(T)$ measured in Ref. 9 for $D=50 \mu \mathrm{m}$ and $\delta l=$ $4.2 \mathrm{~mm}$.

ing spectrometer, resulting in a periodically modulated interference pattern. The intensity $I=I(x)$ of this pattern is recorded by a CCD (Fig. 1b). Here $x$ is the coordinate along the CCD image. The visibility contrast, $V=\left(I_{\max }-I_{\min }\right) /\left(I_{\max }+I_{\min }\right)$, is calculated (Fig. 1 1 ). Finally, a theoretical model that relates $V$ to $\xi_{x}$ is used to analyze the data and determine $\xi_{x}$ as a function of temperature.

The results of this analysis are shown in Fig. 2, As one can see, at $T<4 \mathrm{~K}$ the exciton coherence length grows to a few $\mu \mathrm{m}$, which exceeds the thermal de Broglie wavelength

$$
\lambda_{\mathrm{dB}}=\left(\frac{2 \pi \hbar^{2}}{m k_{B} T}\right)^{1 / 2}
$$

by an order of magnitude $\left(\lambda_{\mathrm{dB}} \sim 0.1 \mu \mathrm{m}\right.$ at $\left.T=2 \mathrm{~K}\right)$. Here $m=0.2$ is the exciton mass in the quantum well in units of the bare electron mass. The inequality $\xi_{x} \gg \lambda_{\mathrm{dB}}$ is anticipated for an exciton system near the superfluid transition. Possibility of such a transition in systems with spatially separated electrons and holes has been put forward in Refs. 12, 13 and 14. The conventional estimate of the transition temperature ${ }^{1,15,16} T_{\mathrm{BKT}} \sim\left(\hbar^{2} / m\right)(n / g)$ gives a few degrees $\mathrm{K}$ for the exciton concentrations ${ }^{17}$ $n / g \sim 10^{10} \mathrm{~cm}^{-2}$ ( $g=4$ is the spin degeneracy).

In Ref. 9 we used an approximation of geometrical optics for describing the light collection in the apparatus. This is justified in the most interesting regime of low $T$ where $\xi_{x}$ is large. On the other hand, at the upper end of the temperature range shown in Fig. 2 the estimated coherence length $\xi_{x}$ was comparable to the diffractionlimited resolution of the optical system, e.g., the Abbe limit 18,19

$$
\mathrm{Ab}=\frac{\lambda_{0}}{2 \mathrm{NA}} .
$$

Here $\mathrm{NA}=\sin \alpha$ is the numerical aperture and $\lambda_{0}$ is the wavelength. In our experiment, $\mathrm{NA}=\sin 16^{\circ} \approx 0.3$ and $\lambda_{0} \approx 800 \mathrm{~nm}$.

In this paper we take diffraction into account and obtain refined estimates of $\xi_{x}$, which are shown in Fig. 2 by the triangles. The difference between previous and current estimates is insignificant for all but a few data points at the boundary of the experimental resolution. Therefore, the case for a rapid and strong onset of the spontaneous coherence of the exciton gas below the temperature of a few degrees $\mathrm{K}$ remains intact.

\section{B. Relation between exciton and optical coherence lengths}

It is well understood that the Abbe limit is not a "hard" limit but simply a characteristic measure of the optical resolution. In fact, another commonly used formulas due to Rayleigh 20,21 differ from Eq. (4) by numerical factors. Roughly speaking, our theoretical model enables us to determine which numerical factor is appropriate for our method of measuring $\xi_{x}$. More precisely, our main result is as follows. Under certain assumptions, $\xi_{x}$ is related to the optical coherence length $\xi$ by

$$
\xi=\sqrt{\xi_{x}^{2}+\frac{1}{Q^{2}}} \quad, \quad \frac{1}{Q}=\frac{\lambda_{0}}{2 \pi \mathrm{NA}} .
$$

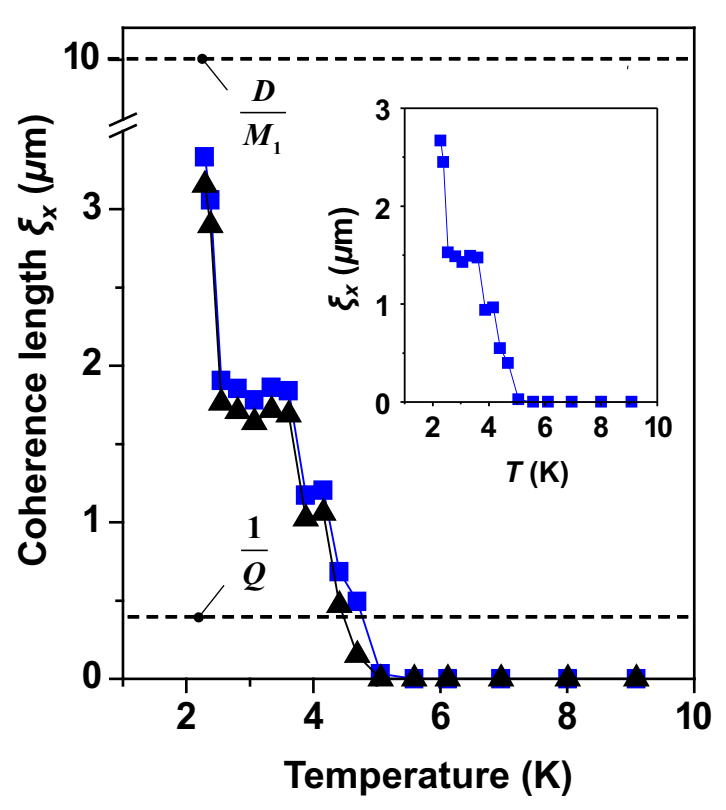

FIG. 2: Main panel: $\xi_{x}$ deduced from the fits of $V(T)$ to the theoretical curves in Fig. 3 The triangles (squares) are the estimates with (without) taking into account the spatial resolution of the experimental setup. The dashed lines indicate boundaries of experimental accuracy. Inset: previous estimates of the coherence length. ${ }^{11}$ 


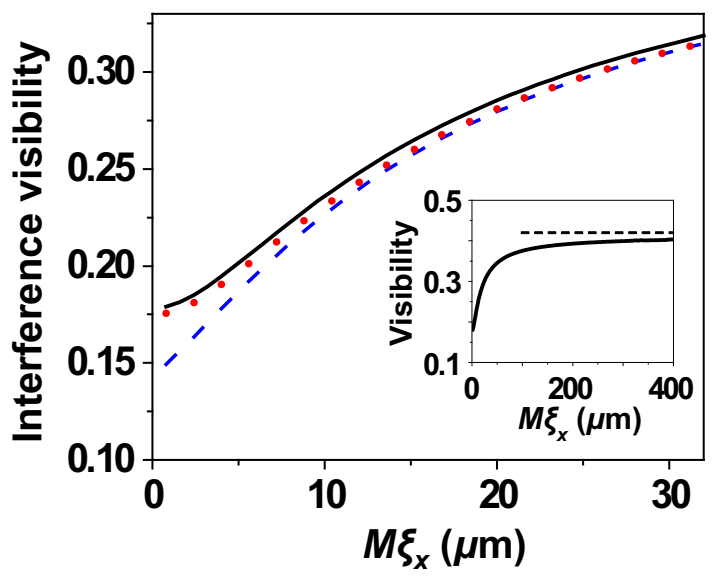

FIG. 3: Visibility of the interference fringes vs. $\xi_{x}$ for parameters $\delta l=4.2 \mathrm{~mm}, D=50 \mu \mathrm{m}, M_{1}=5, M_{2}=1.6$, $Q^{-1}=0.42 \mu \mathrm{m}$. The solid line is the current theory; the dashed line is from Ref. 9; the dotted line is obtained from the dashed one by the replacement $\xi_{x} \rightarrow \sqrt{\xi_{x}^{2}+Q^{-2}}$. The inset shows function $V\left(\xi_{x}\right)$ over a larger range of $\xi_{x}$, with the dashed line indicating the asymptotic value $V\left(\xi_{x}=\infty\right)$.

Here, in analogy to Eq. (2), $\xi$ is defined by

$$
\xi=\frac{1}{M}\left(\int_{0}^{\infty} g(0, R) R d R\right) /\left(\int_{0}^{\infty} g(0, R) d R\right),
$$

where

$$
g(t, R)=\left\langle E\left(t^{\prime}+t, \mathbf{R}^{\prime}+\mathbf{R}\right) E\left(t^{\prime}, \mathbf{R}^{\prime}\right)\right\rangle /\left\langle E^{2}\left(t^{\prime}, \mathbf{R}^{\prime}\right)\right\rangle
$$

is the coherence function 21 of the PL signal $E(t, \mathbf{R})$ emitted by excitons and collected by the described system. In writing this formula we assumed, for convenience, that the second magnification $\left(M_{2}\right)$ of the image occurs before the MZ interferometer, in which case $\mathbf{R}$ and $R=|\mathbf{R}|$ are, respectively, the two-dimensional and the radial coordinates in the plane of the fully magnified image.

Equation (5) is natural because an experimental measurement of any length is affected by the spatial resolution limit. However, Eq. (5) specifically indicates that for $\xi_{x}$ measured using the setup depicted in Fig. 2a, the Abbe limit must be divided by $\pi$. This makes its effect quantitatively smaller than one would naively think.

In addition to the limitation from below, $\xi_{x} \gtrsim 1 / Q$, the accuracy of the present method is restricted from above. When the coherence length exceeds the size of the studied region of the sample $D / M_{1}$, the dependence of $V$ on $\xi_{x}$ should saturate, see the inset of Fig. 3. This may become important at low enough temperatures. The two limitations are indicated by the dashed lines in Fig. 2

\section{GEOMETRICAL OPTICS APPROACH}

In order to construct the model of the described above measurement scheme, we need to know the functional form of $\rho(r)$. Unfortunately, at present there is no comprehensive theoretical framework that provides that. This is because $\rho(r)$ is affected by many factors, including thermal broadening and a variety of scattering mechanisms, see more in Sec. V] On the other hand, the present state of experiment $\underline{\underline{9}}$ does not allow us to extract reliably anything more than a characteristic decay length of function $\rho(r)$. Therefore, we take a phenomenological approach and consider two simple approximations for $\rho(r)$. The first one, used in Ref. 9 , is an exponential

$$
\rho(r)=\rho(0) \exp \left(-\frac{r}{\xi_{x}}\right) .
$$

This ansatz should be reasonable over at least some range of $r$ determined by the interplay of the disorder-limited mean-free path $(\sim 1 \mu \mathrm{m}$ in high-mobility GaAs structures), thermal wavelength $\lambda_{\mathrm{dB}} \sim 0.1 \mu \mathrm{m}$, and possibly, some others. Most importantly, Eq. (8) provides a convenient starting point because it contains a single characteristic length $\xi_{x}$. Later in Sec. IV] we will also consider a Gaussian form,

$$
\rho(r)=\rho(0) \exp \left(-\frac{r^{2}}{\pi \xi_{x}^{2}}\right),
$$

for comparison.

The crucial point is the relation between $\rho(r)$ and $g(R)$. (Here and below we drop $t$ in the argument of $g$ because the time-dependence is not relevant for the calculation.) If the experimental apparatus can be described by geometrical optics, then the only difference between the two functions is the linear magnification $M$ and rescaling by a constant factor. In this case, Eq. (8) entails

$$
g(R)=g(0) \exp \left(-R / M \xi_{x}\right) .
$$

In turn, Eq. (6) gives $\xi=\xi_{x}$, as expected. Until the very end of this section we will use these two lengths interchangeably.

We have shown previously $\frac{9}{\underline{9}}$ that the interference visibility contrast $V$ is related to $g$ as follows:

$$
\begin{gathered}
V=\theta(1-\Delta) V_{0} \\
V_{0}=\frac{\int_{0}^{1} \frac{d z}{z} \sin [F(1-\Delta) z] \sin [F \Delta(1-z)] g\left(z D_{s}\right)}{F \Delta \int_{0}^{1} \frac{d z}{z} \sin (F z)(1-z) g\left(z D_{s}\right)} \\
F=\pi \frac{A N D_{s}}{\lambda_{0}}, \quad D_{s}=M_{2} D, \quad \Delta=\frac{\delta l}{N \lambda_{0}}
\end{gathered}
$$

where $\theta(z)$ is the step-function, $\stackrel{22}{=} A$ is the linear dispersion of the spectrometer, and $N$ is the number of grooves in the diffraction grating. $(A=1.55 \mathrm{~nm} / \mathrm{mm}$ and $N=1.5 \times 10^{4}$ in Ref. 9.)

For $g(r)$ given by Eq. (10) it is straightforward to compute the integrals in Eq. (11); however, in general it has 
to be done numerically. For short coherence lengths, $\xi \ll \lambda_{0} / A N M, D_{s} / M$, one can also derive the analytical formula 9

$$
V \simeq\left(1-\frac{\delta l}{N \lambda_{0}}\right)\left|\frac{\sin \pi X}{\pi X}\right|
$$

where

$$
X=\frac{\delta l}{\delta l_{0}}\left(1-\frac{M}{D_{s}} \xi\right), \quad \delta l_{0}=\frac{\lambda_{0}^{2}}{A D_{s}} .
$$

This equation can be obtained by expanding the sinfactors in the integrals to the order $\mathcal{O}(z)$ and extending their integration limits to infinity.

At $\delta l=\delta l_{0}$ and for small enough $\xi$, Eq. (12) yields

$$
V\left(\delta l_{0}, \xi\right) \simeq\left(1-\frac{\lambda_{0}}{A N D_{s}}\right) \frac{M}{D_{s}} \xi .
$$

Thus, as such $\delta l$ the visibility contrast $V$ vanishes unless $\xi$ is nonzero. Working with $\delta l \approx \delta l_{0}$ ensures the highest sensitivity to $\xi$. In our experiment, $\frac{9}{}$ we extracted $\xi$ at $\delta l=4.2 \mathrm{~mm}$, which is close but not exactly equal to $\delta l_{0}=5.2 \mathrm{~mm}$. Therefore, we computed $V$ using the full formula (11). The results are shown by the dashed line in Fig. 3. We fitted this theoretical curve $V(\xi)$ to the experimentally measured $V(T)$ (Fig. 1 1 ) using $\xi=\xi(T)$ as an adjustable parameter. In this manner we obtain the graph shown by the squares in the main panel of Fig. 2. We see that the exciton coherence length exhibits a dramatic enhancement at $T<4 \mathrm{~K}$. On the other hand, at $T \sim 4 \mathrm{~K}$ this approach gives $\xi_{x}=\xi \sim \lambda_{0}$. One can anticipate that the geometrical optics becomes inaccurate at such small $\xi$, so that $\xi$ and $\xi_{x}$ are in fact different. This question is studied in the next section.

\section{DIFFRACTION EFFECTS}

The conventional theory ${ }^{21}$ of the image formation in optical instruments laid down by $\mathrm{Abbe}^{18}$ in 1873 predicts

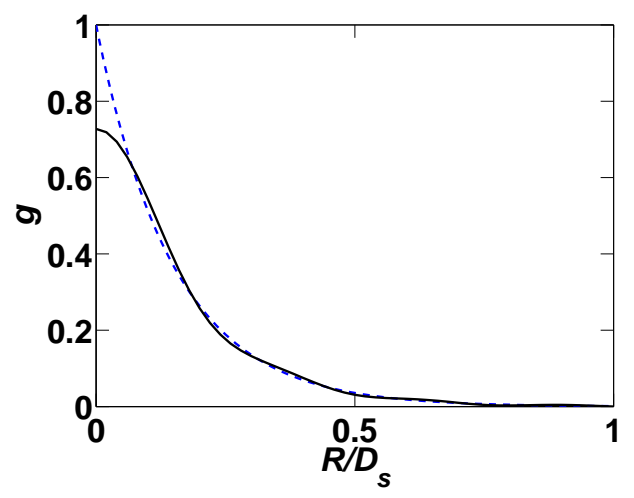

FIG. 4: Optical coherence function $g=g(R)$. The solid line is computed for the following set of parameters: $\xi_{x}=1.5 \mu \mathrm{m}$, $M=8, Q^{-1}=0.42 \mu \mathrm{m}$, and $D_{s}=80 \mu \mathrm{m}$. The dashed line is for geometrical optics, $Q^{-1}=0$. that a point source imaged by a lens with a magnification $M$ creates the diffraction spot

$$
E(R) \propto \int_{k<Q} \frac{d^{2} k}{(2 \pi)^{2}} e^{i \mathbf{k R} / M}=\frac{2 M Q}{R} J_{1}\left(\frac{Q R}{M}\right)
$$

in the image plane. Here $R$ is the radial distance, $Q$ is given by Eq. (5), and $J_{1}(z)$ is the Bessel function. The field distribution (15) is known as the Airy diffraction pattern. ${ }^{23}$ The physical meaning of $Q$ is the largest tangential wavenumber admitted by the lens. Accordingly, the diffraction can be alternatively viewed as a low-pass filtering of the incoming light by the lens. 19

The Airy pattern plays the role of the response function of the lens. Its finite spread in $R$ imposes the limit on the achievable optical resolution $\sim Q^{-1}$ and is the source of the difference between the optical and the actual exciton coherence lengths, see Eq. (5). Indeed, because of the diffraction, the coherence function $g(R)$ is not just a rescaled copy of $\rho(R / M)$ but its convolution with the Airy pattern. Using tilde to denote the 2D Fourier transform, we can express this fact as follows:

$$
\tilde{g}(k) \propto \theta(Q-M k) \tilde{\rho}(M k) .
$$

Note that $\tilde{\rho}(k)$ has the physical meaning of the momentum distribution function for excitons. Computing $\tilde{\rho}(k)$ from Eq. (8), we get

$$
\tilde{g}(k)=\text { const } \times \frac{\theta(Q-M k)}{\left(1+M^{2} \xi_{x}^{2} k^{2}\right)^{3 / 2}} .
$$

The constant prefactor in this formula has no effect on $V$. It is convenient to choose it to be $2 \pi M^{2} \xi_{x}^{2}$, so that

$$
g(R)=M^{2} \xi_{x}^{2} \int_{0}^{Q / M} \frac{J_{0}(k R) k d k}{\left(1+M^{2} \xi_{x}^{2} k^{2}\right)^{3 / 2}} .
$$

In this case in the limit $Q \rightarrow \infty$, we recover Eq. (10) with $g(0)=1$. On the other hand, for finite $Q$, we have

$$
g(0)=1-\frac{1}{\sqrt{1+Q^{2} \xi_{x}^{2}}} .
$$

Additionally, at large $R$, function $g(R)$ acquires the behavior characteristic of the Airy pattern (15): quasiperiodic oscillations with the envelope decaying as $R^{-3 / 2}$, see Fig. 4. Finally, computing the optical coherence length $\xi$ according to Eq. (6) we get Eq. (5).

The refined theoretical dependence of $V$ on $\xi_{x}$ can now be obtained by substituting Eq. (18) into Eq. (11). As before, for small $\xi_{x}$ analytical formulas (12)-(14) suffice, with $\xi$ defined by Eq. (5). When this $\xi$ becomes comparable to $D_{s} / M$, numerical evaluation of Eqs. (11) and (18) is necessary. The representative results are shown by the solid line in Fig. 3. For comparison, two other curves are included. The dashed line is the geometrical optics approximation, $\xi=\xi_{x}$ of Sec. III. The dotted line 
is the result of correcting the latter according to Eq. (5) and using $Q$ appropriate for our experiment. As one can see, at small $\xi_{x}$, the effect of the diffraction-limited resolution of the optical system is indeed accounted for by Eq. (5). At large $\xi_{x}$, the correction becomes small and all the curves are very close to each other.

It is instructive to examine how our conclusions so far depend on the model assumption (8) about function $\rho(r)$. To this end we consider next the Gaussian ansatz (9), which is similar to Maxwell-Boltzmann distribution except the coefficient in the exponential factor is adjusted to satisfy Eq. (2). Let us compute the corresponding $\xi$. Using Eq. (16), we can rewrite Eq. (6) as

$$
\xi=\tilde{\rho}(0) /\left(\int_{0}^{Q} d k \tilde{\rho}(k)\right) .
$$

Substituting here

$$
\tilde{\rho}(k)=\tilde{\rho}(0) \exp \left(-\frac{\pi}{4} k^{2} \xi_{x}^{2}\right),
$$

which follows from Eq. (9), we get

$$
\xi=\frac{\xi_{x}}{\operatorname{erf}\left[(\sqrt{\pi} / 2) Q \xi_{x}\right]},
$$

where $\operatorname{erf}(z)$ is the error function. This formula replaces Eq. (5). Interestingly, it implies that for the same $\xi_{x}$ and $Q$, the effect of the finite resolution in the case of a Gaussian decay is always smaller than for the exponential one. The direct numerical evaluation of Eq. (11) with the Gaussian profile (9) confirms this expectation, see Fig. 5. Thus, we again conclude that the diffraction correction is important for $\xi \lesssim \lambda_{0}$, but it is very small in the most interesting region $\xi>\lambda_{0}$.

Notice that function $V\left(\xi_{x}\right)$ increases somewhat faster with $\xi_{x}$ for the Gaussian case compared to the exponential one, cf. Figs. 3 vs. 5 . Therefore, had we adopted the Gaussian ansatz (9), the deduced values of $\xi_{x}(T)$ would have been somewhat smaller than those plotted in Fig. 2 . This is to be expected: if the exact functional form of $\rho(r)$ is unknown, its characteristic decay length can be determined only up to a numerical coefficient of the order of unity.

\section{DISCUSSION}

The main purpose of the present work is refinement of the optical method for determining the exciton coherence length $\xi_{x}$. Unlike previously proposed schemes,,$+\frac{7.8}{.}$ which involve angle-resolved photoluminescence, our technique is based on real-space interferometry.

We showed that in order to obtain an accurate estimate of $\xi_{x}$, the optical coherence length $\xi$ of the exciton emission should be corrected because of the diffraction-limited spatial resolution of the experimental apparatus. However the correction is insignificant as long as $\xi_{x}$ is larger than about one wavelength and the numerical aperture

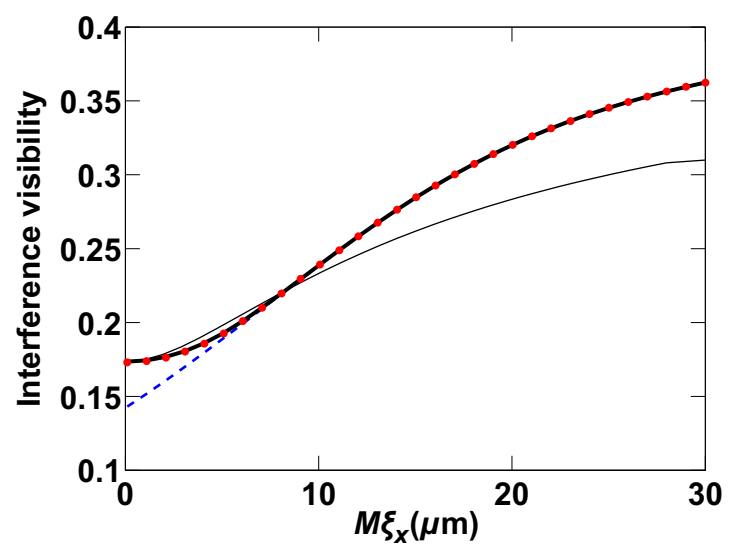

FIG. 5: Visibility of the interference fringes vs. $\xi_{x}$ for the Gaussian coherence function (9) and the parameters of Fig. 3 The thick solid line includes the diffraction correction; the dashed line is for the geometrical optics; the dotted line is obtained from the dashed one by the replacement of $\xi_{x}$ by $\xi$ according to Eq. (22). The thin solid line is $V\left(\xi_{x}\right)$ for the exponential ansatz, replotted from Fig. 3 to facilitate the comparison.

NA of the experimental setup is not too small. The correction does grow as NA decreases, and so reduced NA should be avoided.

It is well known $18,19,21$ that limitation of the spatial resolution due to diffraction is equivalent to that due to restriction on tangential wavenumbers $k$ admitted by the lens collecting the signal. This $k$-filtering effect has been considered in Ref. 24 in application to the measurements of the exciton coherence length. For the collection angle $\alpha=16^{\circ}$ in our experiments,,$\underline{9}$ the results presented in Fig. 3c of Ref. 24 give the correlation length due to the $k$-filtering effect $\xi_{\gamma} \approx 1 \mu \mathrm{m}$. (This length plays the role similar to that of $Q^{-1}=0.42 \mu \mathrm{m}$ in our formalism.) For the considered $\rho(r)$ this correction enters either through the quadratic sum, Eq. (5), or the error function, Eq. (22). As a result, the estimation of the $k$-filtering effect per Ref. 24 gives only a small $(\sim 10 \%)$ correction, e.g., $\xi-\sqrt{\xi^{2}-\xi_{\gamma}^{2}}$ to the optical coherence length $\xi$ measured in Ref. 9 at low $T$. Therefore, it cannot explain the observed large enhancement of the coherence length at $T<4 \mathrm{~K}$. Our calculations indicate that the correction is even smaller.

The discussion of physics that is responsible for the observed rapid change in $\xi_{x}$ at low temperatures is however beyond the score of this paper. As a final word, we would like to offer only the following minimal remarks on this matter.

The density matrix $\rho(r)$, from which $\xi_{x}$ is defined through Eq. (2), is influenced by a number of factors, including Bose statistics, interactions, and scattering. The effect of the first two has been studied extensively, albeit for simplified models of interaction. According to present understanding, $\stackrel{1,2}{\stackrel{2}{2}}$ the long-distance behavior 
of function $\rho(r)$ is qualitatively different above and below the Berezinskii-Kosterlitz-Thouless (BKT) transition temperature $T_{\mathrm{BKT}}$. At $T \gg T_{\mathrm{BKT}}$, where $\tilde{\rho}(k)$ obeys the classical Boltzmann statistics, $\rho(r)$ decays as a Gaussian, Eq. (9), with the coherence length

$$
\xi_{x}=\lambda_{\mathrm{dB}} / \pi .
$$

[Our estimates of $\xi_{x}$ at $T<4 \mathrm{~K}$ exceed $\lambda_{\mathrm{dB}} / \pi$ by an order of magnitude, suggesting that Eq. (223) does not apply at such temperatures.] At $T<T_{\mathrm{BKT}}$, the eventual asymptotic decay of the density matrix becomes algebraic, $\rho(r) \propto r^{-\nu}$ with a temperature-dependent exponent $\nu(T)$. The behavior of $\rho(r)$ at intermediate temperatures and/or distances is more complicated. In general, it can be computed only numerically, e.g., by quantum Monte-Carlo method $\stackrel{2}{2}$

Some of the other mechanisms of limiting the coherence length $\xi_{x}$, such as exciton recombination and exciton-phonon scattering are too weak to significantly affect the large magnitude of observed $\xi_{x}$ at low temperatures $\stackrel{9}{=}$ However, scattering by impurities and de- fects should be seriously considered. It can substantially modify the functional form of $\rho(r)$ compared to the disorder-free case. Indeed, weak disorder typically leads to an exponential decay of the correlation functions on the scale of the mean-free path, which in fact inspired our ansatz (8). As temperature goes down, the strength of the disorder decreases because excitons can screen it more efficiently, $25,26,30$ This should increase both the mean-free path and the exciton coherence length.

The comprehensive theoretical calculation of the exciton coherence length that would take into account all relevant thermal, interaction, and disorder screening effects is yet unavailable.

\section{Acknowledgments}

We thank A. Ivanov and L. Mouchliadis for valuable discussions and comments on the manuscript. This work is supported by NSF grants DMR-0606543, DMR0706654, and ARO grant W911NF-05-1-0527.
1 V. N. Popov, Functional Integrals in Quantum Field Theory and Statistical Physics (Reidel, Dordrecht, 1983).

2 Yu. Kagan, V. A. Kashurnikov, A. V. Krasavin, N. V. Prokof'ev, and B. V. Svistunov, Phys. Rev. A 61, 043608 (2000).

3 For review, see L. V. Butov, J. Phys.: Condens. Matter 16, R1577 (2004).

4 Th. Östereich, T. Portengen, and L. J. Sham, Solid State Commun. 100, 325 (1996).

5 B. Laikhtman, Europhys. Lett. 43, 53 (1998).

${ }^{6}$ A. Olaya-Castro, F. J. Rodriguez, L. Quiroga, and C. Tejedor, Phys. Rev. Lett. 87, 246403 (2001).

7 J. Keeling, L. S. Levitov, and P. B. Littlewood, Phys. Rev. Lett. 92, 176402 (2004).

8 R. Zimmermann, Solid State Commun. 134, 43 (2005).

9 S. Yang, A. T. Hammack, M. M. Fogler, L. V. Butov, and A. C. Gossard, Phys. Rev. Lett. 97, 187402 (2006).

10 L. V. Butov, A. C. Gossard, and D. S. Chemla, arXiv:cond-mat/0204482 Nature (London) 418, 751 (2002).

11 Here we remedy an error in handling parameter $M_{2}$ in the previous paper $\stackrel{9}{-}$ Therein $M_{2}=1.6$ was determined from the data analysis. However, by confusion the estimated value of $M \xi$ was divided by $M=10$ (instead of $M=5 \times$ $1.6=8$ ) to give the result for $\xi$. Accordingly, the values of $\xi$ in Fig. 3e of Ref. 9 are somewhat smaller than what should have been shown. (For ease of comparison, this figure is reproduced in the inset of Fig. 2) In the main panel of Fig. 2 and the rest of the present paper this error has been corrected: we use $M_{2}=1.6$ and $M=8$ throughout.

12 Yu. E. Lozovik and V. I. Yudson, Pis'ma Zh. Eksp. Teor. Fiz. 22, 556 (1975) [JETP Lett. 22, 274 (1975)].

13 S. I. Shevchenko, Fiz. Nizk. Temp. 2, 505 (1976) [Sov. J. Low Temp. Phys. 2, 251 (1976)].

14 T. Fukuzawa, S. S. Kano, T. K. Gustafson, and T. Ogawa, Surf. Sci. 228, 482 (1990).
15 D. S. Fisher and P. C. Hohenberg, Phys. Rev. B 37, 4936 (1988).

16 N. Prokof'ev and B. Svistunov, Phys. Rev. A 66, 043608 (2002).

17 Exciton concentration $n$ can be deduced from the exciton energy $E_{x}=h c / \lambda_{0}$ using the linear relation $n=$ $\left(C / e^{2}\right)\left[E_{x}-E_{x}(n=0)\right]$. However, the value of the coefficient $C$ is presently under debate. Earlier literature ${ }^{27,28,29,30}$ assumed that $C$ is equal to the classical capacitance per unit area $C=\epsilon /(4 \pi d)$, where $\epsilon$ is the dielectric constant and $d$ is the separation between the electron and hole layers. A more recent calculation ${ }^{31}$ gives an order of magnitude higher estimate for $C$ and therefore $n$ in our experiments.

18 E. Abbe, Arch. f. Mikroskop. Anat. 9, 413 (1873).

19 H. Köhler, J. Mod. Opt. 28, 1691 (1981).

20 L. Rayleigh, Mon. Notes Roy. Astron. Soc. 3359 (1872). Reprinted in L. Rayleigh, Scientific Papers (Dover, New York, 1964), Vol. I, p. 163.

21 M. Born and E. Wolf, Principles of Optics, 7th ed. (Cambridge University Press, Cambridge, UK, 1999).

22 The absence of " $\theta$ " and the extra "sin" in the corresponding Eq. (7) of Ref. 9 are typographic errors.

23 G. B. Airy, Phil. Mag. Ser. 3 18, 1 (1841). The Airy formula is actually not exact but it is a good approximation near the optical axis, see J. D. Jackson, Classical Electrodynamics (Wiley, New York, 1998).

24 L. Mouchliadis and A. L. Ivanov, arXiv:0802.4454

25 L. V. Butov and A. I. Filin, Phys. Rev. B 58, 1980 (1998).

${ }^{26}$ D. E. Nikonov and A. Imamoglu, ArXiv:quant-ph/9806003.

27 D. Yoshioka and A. H. MacDonald, J. Phys. Soc. Jpn. 59, 4211 (1990).

28 X. Zhu, P. B. Littlewood, M. Hybertsen, and T. M. Rice, Phys. Rev. Lett. 74, 1633 (1995).

${ }^{29}$ Yu. E. Lozovik and O. L. Berman, JETP Lett. 64, 573 
(1996).

30 A. L. Ivanov, Europhys. Lett. 59, 586 (2002).
${ }^{31}$ C. Schindler and R. Zimmermann, arXiv:0802.3337 